\title{
Neurostimulation Article Clarification
}

\section{TO THE EDITOR:}

We would like to sincerely apologize for the errors published in our article in the October/November issue of Pain Physician, "Occipital Neurostimulation-induced Muscle Spasms: Implications for Lead Placement" (1). Though an explanation appeared in the November/December issue, the information reported below is more complete and supersedes the previous erratum:

The main focus of the case report was that leads placed at the $\mathrm{C} 1$ level can result in muscle recruitment and muscle spasm. Leads at or above the nuchal ridge do not cause the same problem as shown by the case revisions reported here. This is very important information and it has nothing to do with the manufacturer of the neurostimulation devices. We note the manufacturer only because it was a reviewer request/journal policy. In the body of the report we made 2 errors that we wish to correct. In Case 4, we misnamed the St. Jude Eon device, which was actually a dual Octrode ANS Renew device, and we misnamed the Medtronic Restore Ultra, which was actually a single quadripolar Medtronic lead (Quad Plus) with Itrel 3 device. The second error was with the $\mathrm{x}$-rays in Fig. 4 referenced in the same case. The $x$-rays are not those of the patient described in the case, but are representative of lead [mal]placement. Therefore, the case should have noted that Fig. 4 was an example of the lead position at C1 of a single 4 contact lead. The case was otherwise accurate and the revision of the lead to a location above the nuchal ridge resolved the patient's problem of spasm and muscle recruitment.

The authors regret any confusion or misunderstanding based on these errors and hope the reader takes home the major point, that this is a clinical issue of lead placement and has nothing to do with lead or generator failure, or product function. The reader should not make any conclusions about specific devices based on this paper, but should have a better understanding of the surgical technique for occipital neurostimulation.

Salim Hayek, MD, PhD

Associate Professor, Dept of Anesthesiology

Case Western Reserve University

Chief, Division of Pain Medicine

University Hospitals

Cleveland, $\mathrm{OH}$

E-mail: Salim.hayek@UHhospitals.org

Joseph F. Jasper, MD

Advanced Pain Medicine Physicians

Tacoma, WA

Timothy R. Deer, MD

The Center For Pain Relief

Charleston, WV

Samer N. Narouze, MD

Department of Pain Management

Cleveland Clinic Foundation

Cleveland, $\mathrm{OH}$

\section{References}

1. Hayek S, Jasper JF, Deer TR, Narouze SN. Occipital neurostimulation-induced muscle spasms: Implications for lead placement. Pain Physician 2009; 12;867-876. 


\section{Herpes Zoster: Are Selective Nerve Root Injections the Treatment or the Cause?}

\section{TO THE EDITOR:}

We read with great interest the case report "Herpes Zoster Radiculopathy Treated with Fluoroscopically-Guided Selective Nerve Root Injection" (1) as we recently encountered 2 cases of reactivation of herpes zoster after selective nerve root injection in a patient with transforaminal stenosis.

In the first case, a 30-year-old female presented to our pain clinic with one year of low back pain with radicular symptoms involving the right leg and foot. $A$ magnetic resonance imaging (MRI) scan of the patient showed a herniated nucleus pulposus at L3-L4 and L4-L5 with bilateral transforaminal stenosis at L4-L5. Physical examination revealed tenderness in the lower lumbar spine and a positive straight leg raise. With patient approval, a bilateral transforaminal epidural steroid injection was performed at L4 level under fluoroscopic guidance. AP and lateral images were obtained to verify the position of the needle within the safe triangle area. Non-ionic contrast injection of $0.4 \mathrm{~mL}$ produced a neurogram (Fig. 1). After confirmation of safe needle location, $40 \mathrm{mg}$ of triamcinolone acetonide in $1 \mathrm{~mL}$ of $2 \%$ lignocaine was injected on each side. Five days following the transforaminal injection, the patient developed a burning sensation in the right T10 dermatome, which was rapidly followed by the emergence of classic herpes zoster lesions (Fig. 2). She was started on acyclovir 800 mg 5 times daily for 7 days, whereupon the lesion resolved and post-herpetic neuralgia did not occur.

In another case, a 42-year-old female patient presented with radiculopathy of 6 months duration. MRI showed a herniated nucleus pulposus at L5-S1 with transforaminal stenosis. Physical examination revealed a positive straight leg raise. Transforaminal steroid injection was performed on right side at $L 5$ level under fluoroscopic guidance and $40 \mathrm{mg}$ of triamcinolone acetonide in $1 \mathrm{~mL}$ of $2 \%$ lignocaine was injected 3 consecutive times at an intervals of 3 weeks. The patient had significant relief in her radicular symptoms. However, after the third injection, the patient developed some pruritis in her right hypochondrial region. Examination revealed skin changes typical of a herpes zoster outbreak in T8 distribution. After consultation with a der-
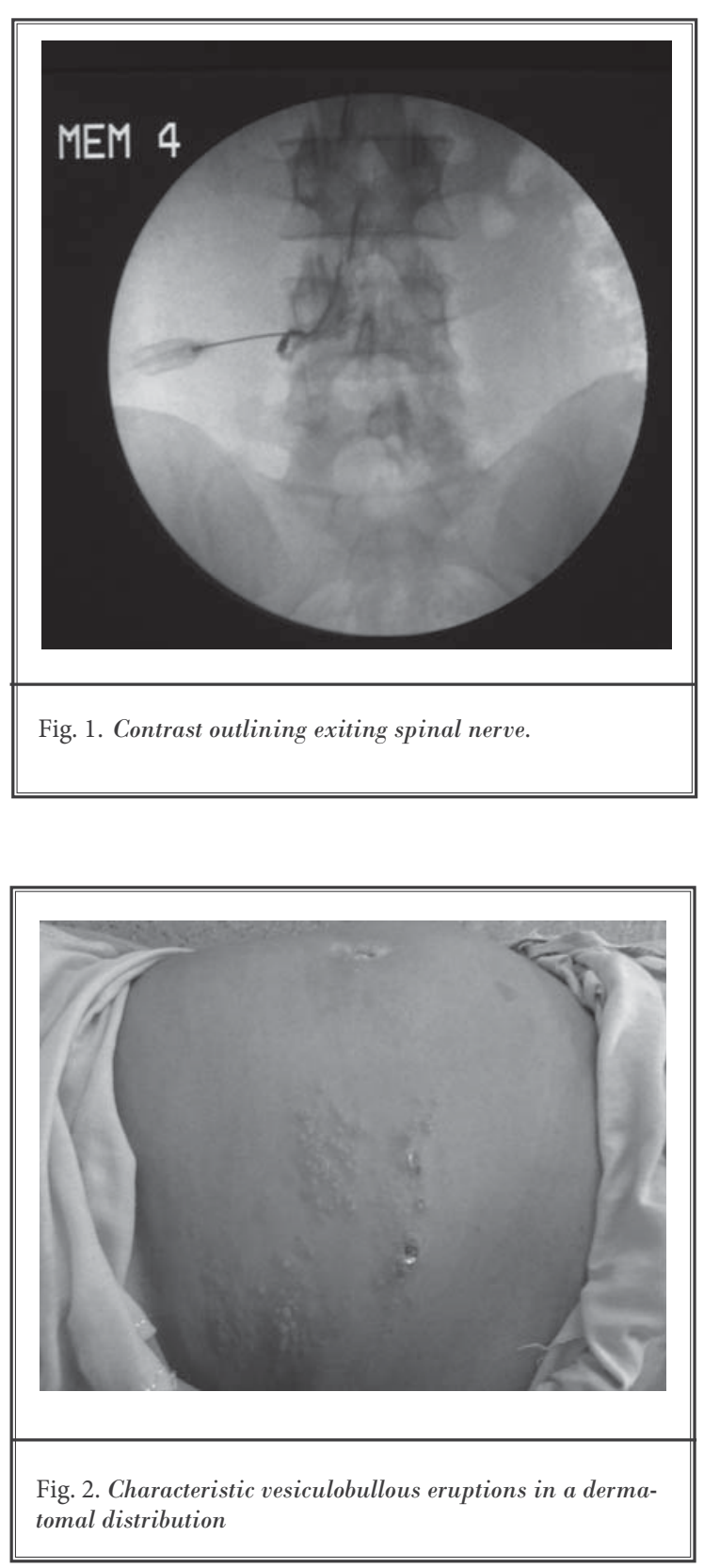
matologist, she was started on a course of oral acyclovir. Amitryptiline $10 \mathrm{mg}$ daily was added to prevent post-herpetic neuralgia. Over the next few days, the skin lesions crusted, and eventually the skin returned to its normal condition. No post-herpetic neuralgia developed.

Epidural steroids are often used during treatment of herpes zoster and post herpetic neuralgia (2). Rationale for their use is to help suppress inflammation that could be aggravating and increasing pain. However, the use of epidural steroids has also been implicated in herpes zoster activation in 2 different case reports $(3,4)$. Szokol and Gilbert (3) reported a case of a 53 -year-old female who developed herpes zoster in the 53 distribution after $120 \mathrm{mg}$ of methylprednisolone epidural injection for right sided radiculopathy. Four days later, patient developed typical lesions of herpes zoster in S3 distribution (3). Parsons et al (4) described a 42-year-old man who sustained injury to his right foot from a fall, underwent multiple surgeries, and culminated in a triple arthrodesis. He then developed complex regional pain syndrome limiting weight bearing and ambulation. Because of persistent pain after oral medications and Bier's block, patient was scheduled for serial epidural blocks with methylprednisolone. One week after the fifth epidural injection, the patient developed burning pain in the right L2 dermatome followed by development of classical herpes zoster rash. Both these reports describe a close temporal association between an epidural steroid injection and development of acute herpes zoster.

Our patients developed skin changes typical of a herpes zoster outbreak shortly after transforaminal steroid injections. Microscopic examination revealed characteristic multinucleated keratinocytes with nuclear molding and margination of chromatin. Herpes simplex virus 1 and 2 infection was ruled out through evaluation by PCR.

Our cases demonstrate a possible link between the use of transforaminal steroids and reactivation of herpes zoster. As the eruptions involved dermatomes distant from the site of injection, it can be postulated that glucocorticoids might have caused hypothalamic-pituitary axis (HPA) suppression by acting directly on central glucocorticoid receptors, presumably via CSF absorption
(5). Injection of corticosteroid into the intervertebral foramen and segmental nerve canal allows for a concentrated application of steroids around the inflamed neural structures. However, this advantage comes with increased complexity and brings with it increased risk of damage to spinal nerve or dorsal root ganglion (DRG) from direct needle trauma (6). So, another possibility is that localized trauma to the nerve root/ DRG may have precipitated the herpetic eruption $(7,8)$. Further, since both cases presented at an interval of 2 months, we believe the probability of both patients being in the prodromal phase of a shingles outbreak while undergoing an epidural steroid injection is remote.

The case report of Conliffe et al (1) and our 2 cases leave us perplexed; can the same transforaminal steroid injection be the causative factor and at the same time used as a treatment modality for herpes zoster? Also is it right to conclude that transforaminal epidural injection or selective nerve root block may be an effective treatment for zoster motor paresis based on a single care report?

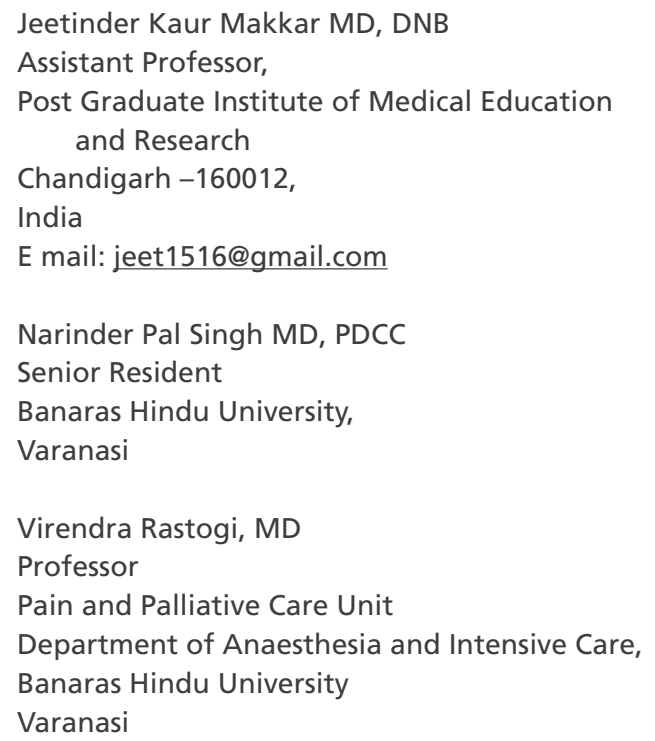

\section{References}

1. Conliffe TD, Dholakia M, Broyer Z. Herpes zoster radiculopathy treated with fluoroscopically guided selective nerve root injection. Pain Physician 2009; 12: 851-853.

2. Postherpetic neuralgia involving the right $C_{5}$ dermatome treated with a cervical transforaminal epidural steroid injection: A case report. Arch Phys Med 4 Rehabil 2007; 88:255-258.

3. Szokol JW, Gilbert HC. A herpes zoster outbreak temporarily associated with an epidural steroid injection. Reg Anes th Pain Med 1998; 23:328.

. Parsons SJ, Hawboldt GS. Herpes zoster: A previously unrecognized complication of epidural steroids in the treatment of complex regional pain syn- 
drome. J Pain Symptom Manage 2003; 25:198-199.

5. Jacobs S, Pullan PT, Potter JM. Adrenal suppression following extradural steroids. Anaesthesia 1983; 38:953-956.

6. Landers MH, Jones RL, Rosenthal RM. Lumbar spinal neuroaxial procedures.
Interventional Pain Management: Image Guided Procedures. Raj PP(Ed) Saunders Elsevier, Philadelphia, 2008, 8. 322-367.

7. Svozilkova P, Rihova E, Diblik P, Kuthan P, Kovarik Z, Kalvodova B. Varicella zoster virus acute retinal necrosis follow- ing eye contusion: Case report. Virol J 2005; 2:77.

Lee MR, Ryman W. Herpes zoster following cryosurgery. Australas I Dermatol $2005 ; 46: 42-43$.

\section{In response}

I am writing in response to the interesting case report entitled, "Herpes Zoster: Are Selective Nerve Root Injections the Treatment or the Cause," authored by Makkar et al in the most recent issue of Pain Physician.

In our case report we treated a patient with herpes zoster radiculopathy with fluoroscopically guided selective nerve root injections on the right at the L5 spinal level. The patient had clinically confirmed zoster eruption in the lumbar spine well before he came to our clinic. He had not been treated with steroids. The patient developed a foot drop that was confirmed by electrodiagnosis as an L5 radiculopathy, without evidence of peripheral neuropathy. After 4 weeks of conservative treatment, we performed 2 right $L 5$ fluoroscopically guided nerve root injections 2 weeks apart and not only did our patient's pain improve but he also had a return of motor function.

Our case and a similar case of cervical radiculopathy, published by Shakir et al (1) in 2007, demonstrate efficacy with epidural steroid injections at the affected spinal level in treatment of herpes zoster radiculopathy. We offer these injections as a potential treatment in this select group of patients after other conservative measures such as antiviral medications, physical therapy, and analgesic medications have failed. We also acknowledge that we could not exclude other spinal causes for our patient's symptoms and signs.
Conversely, the cases presented by Dr. Makkar's group developed reactivation of herpes zoster after epidural steroid injection. A more plausible explanation which was correctly suggested by their group is that the patient's immune system was suppressed by concentrated dosage of steroids in these susceptible patients.

Our group would argue that any immunosuppressant medications such as immuran, methotrexate, and oral steroids would have contributed to a similar outcome in Makkar's patients.

Thank you for the opportunity to respond to this interesting case report.

Theodore D. Conliffe, Jr., MD

Clinical Asst. Professor

Dept. of Physical Medicine and Rehabilitation

Thomas Jefferson University Hospital

Rothman Institute of Orthopedics

Philadelphia, PA

Zach Broyer, MD

Madhuria Dholakia, MD

Rothman Institute of Orthopedics

Philadelphia, PA

\section{Reference}

1. Shakir A, Kimbrough DA, Mehta B. Post herpetic neuralgia involving the right $\mathrm{C}_{5}$ dermatome treated with a cervical transforaminal epidural steroid injection. a case report. Arch Phys Med Rehab 2007; 88:255-258. 